\title{
CONSIDERAZIONI PRAGMALINGUISTICHE SU TRE DISCORSI DI POLITICI CROATI
}

\author{
Rita Scotti Jurić \\ Università degli Studi Juraj Dobrila di Pola (Croazia) \\ rscotti@unipu.hr \\ Renata Pokrajac \\ shena102@hotmail.it
}

\section{Riassunto}

Il discorso della politica, pur essendo indirizzato verso l'immediatezza, la semplicità e la chiarezza, in effetti si nasconde dietro a concetti generici e luoghi comuni. Appare spesso oscuro, vago, ambiguo, autoreferenziale. Le ricerche pragmalinguistiche individuano in questi discorsi informazioni rilevanti relative a tutti quegli elementi impliciti del contesto e del co-testo. In questa ricerca sono stati analizzati e discussi i discorsi di tre personaggi della politica croata e segnalate le similitudini e le differenze tramite l'analisi dei deittici, delle presupposizioni, delle implicature e degli atti linguistici individuati.

Parole chiave: pragmalinguistica, linguaggio della politica, atti linguistici, implicature conversazionali, deissi 


\section{Il linguaggio della politica}

Le ricerche sul linguaggio della politica non sono poche (Stanyer, 2007; Marletti, 2010; Giansante, 2011; Campus, 2010; Dell'Anna, 2010; Cortelazzo, 2016; Desideri, 1983, 1998, 1998b), ma quello del linguaggio politico rimane comunque un campo ancora poco studiato, soprattutto in prospettiva pragmalinguistica. La politica "è il regno della parola, non dei fatti" dice Ruggiano (2013) e, "come in teatro, il politico ha davanti a sé un pubblico che deve portare dalla sua parte raccontando una storia convincente ed emozionante" $(2013,223)$. Per comprendere il discorso politico, si dovrebbe sottoporlo all'analisi del contesto storico-culturale nel quale nasce, al momento in cui viene pronunciato e all'uditorio al quale è diretto (Cedroni, 2014). Il linguaggio politico ha come prima funzione quella di convincere presentando come vera un'interpretazione dei fatti e facendo riferimento ai valori, ai turbamenti o alle emozioni presenti nel pubblico che ascolta. Queste intenzioni si riversano e prendono corpo attraverso il linguaggio usato, in quanto "la lingua non è, e non sarà mai, uno strumento lineare e non-ambiguo." Tutt’altro - "la lingua è il flusso sanguigno della politica" e "il potere politico viene esercitato innanzitutto attraverso il linguaggio. “[1] Saranno, appunto, l'esemplificazione retorica e l'uso di uno stile colloquiale gli elementi maggiormente usati in questo format della comunicazione orale (Wodak, 2009; Van Dijk, 2005). Il parlare di sé (self-presentation) e il riferire notizie confidenziali (selfdisclosure) saranno gli elementi costanti in numerosi script comunicativi di tipo politico (Schlenker, 1980; Gilbert, Cambon, 2003; Archer, Cook, 1986). Si tratta di narrazioni di contenuto intimo, privato, rivolte dall'oratore a un pubblico eterogeneo di uditori con cui non intrattiene rapporti di natura personale, ma con i quali intende costruirne avendo specifici intenti persuasivi. L'obiettivo è quello di aumentare la propria credibilità, ridurre le distanze, creare più un'impressione positiva di se stessi. Ovviamente, i politici che usano queste strategie linguistiche, sono generalmente percepiti come amichevoli, aperti e riescono a guadagnare celebrità e consenso (Collins, Miller, 1994). Bongelli, Riccioni et alii (2012)

[1] Daša Drndić, Prilagođavanje Riječi: Jezik i Politika, Sarajevske Sveske br. 10, in: http://www.sveske.ba/en/ content/prilagodavanje-rijeci-jezik-i-politika. Traduzione delle autrici. 
ricordano che i parlanti cercano spesso di mettere in campo la simpatia, lo humour, l'autoironia, e fanno spesso riferimenti a valori importanti quali la famiglia, la patria, la libertà o la religione. Alle volte il linguaggio dei politici appare oscuro, vago, ambiguo, autoreferenziale e si nasconde dietro a concetti generici e luoghi comuni. Sembra che sia "un parlare per dire," un parlato vuoto di significati e pieno di ambiguità linguistiche. C'è chi comunque desidera avere interazioni con i propri interlocutori come tra il gruppo di pari, in maniera dialogica, cercando di stabilire una relazione vera (Spina, 2012). Lasswell e Fox (1979) ${ }^{[2]}$ ritengono che il linguaggio della politica voglia sviluppare potere e fornire decisioni attraverso un esercizio di persuasione, una negoziazione verbale per instaurare un rapporto di confidenza con il pubblico, esponendo progetti futuri o su fatti attuali e volgendosi agli errori o successi del passato. Per giungere a tanto darà un forte valore illocutivo ai suoi enunciati usando un lessico eufemizzante, perspicuo, che maschera i fatti e le opinioni e veicola implicature allusive. L'"oratore di professione", anche se mantiene una certa formalità nell'eloquio, non riesce a celare elementi che rivelano inferenze e impliciti di vario tipo. Il più delle volte se l'informalità viene a eccedere, si potrà pensare che sia dovuta al raggiungimento di uno scopo prefissato.

\section{Finalità, soggetti e metodo}

La presente ricerca vuole individuare, con un'analisi quantitativa, gli elementi pragmalinguistici maggiormente usati dai personaggi politici nell'atto della stesura dei propri discorsi in pubblico e rilevare, mediante un'analisi qualitativa, le differenze individuali dei singoli parlanti, nonché quelle comuni a questo genere discorsivo. Nella ricerca abbiamo preso in considerazione il linguaggio di tre personaggi della politica odierna, Milan Kujundžić, Milan Bandić e Ivo Josipović. La scelta è stata puramente casuale e confluiva con le esercitazioni di Pragmalinguistica presso la Facoltà di Studi Interdisciplinari, Italiani e Culturali di Pola. Il corpus, preso da You tube ${ }^{[3]}$, della durata che variava dai $2 \mathrm{~min}$. e $21 \mathrm{sec}$.

[2] https://www.sbt.ti.ch/dep/bclu/lingua.pdf

[3] Kujundžić: https://www.youtube.com/watch?v=Xs7G1RY_Qy4 Durata: 02:49 minuti. Consultato il 20.05.2017. Bandić: https://www.youtube.com/watch?v=NvanBlYgF54 Durata: 02:23minuti. Consultato il 20.05.2017. Josipović:https://www.youtube.com/watch? $\mathrm{v}=\mathrm{n} 23 \mathrm{o} 2 \mathrm{KmUuwY}$ Durata: 02:39 minuti. Consultato il 20.05.2017. 
ai 2 min. e $49 \mathrm{sec}$ per soggetto, è stato regolarmente trascritto e analizzato. Siamo partiti dall'ipotesi che l'informalità linguistica dei politici potrebbe offrire indicazioni significative per una lettura dei linguaggi su uno sfondo pragmalinguistico. Il discorso di Josipović e quello di Kujundžić appartengono alla candidatura alle elezioni presidenziali della Croazia nel novembre-dicembre 2014, mentre quello di Bandić è stato fatto dopo la vittoria all'elezione del sindaco della città di Zagabria. Per quanto il corpus registrato sia di una decina di minuti circa, in questa ricerca pilota possiamo comunque constatare che tutti e tre i discorsi sono simili tra di loro per la forma, ma ci sono delle differenze sul piano della formalità/ informalità.

\section{Discussione dei dati}

3.1. Il discorso dei politici, come del resto quello tutti gli altri, dietro a un'apparente esplicitazione dei fatti, nasconde un pensiero implicato, inferito. La finalità principale di questa ricerca sarà quella di dare un'interpretazione inferenziale quanto più corretta possibile. In questo senso, i primi elementi che rendono la frase incomprensibile a persone esterne al contesto, o poco attenti al co-testo, sono le deissi, le quali rappresentano il modo più evidente in cui la relazione tra lingua e contesto è riflessa nella struttura delle lingue (Levinson, 1985; Yule, 1986; Papi, 2000). Le deissi nei discorsi analizzati (di persona, spaziale, temporale, del discorso e la deissi sociale $)^{[4]}$ non si differenziano solamente da parlante a parlante, ma anche in base alla situazione.

Tabella 1. Deissi nel linguaggio dei tre partecipanti

\begin{tabular}{|c|c|c|c|c|c|c|c|c|c|}
\hline & \multicolumn{2}{|c|}{ D. persona } & \multicolumn{2}{c|}{ D. spaziale } & \multicolumn{2}{c|}{ D. temporale } & \multicolumn{2}{c|}{ D. discorso } & Tot. \\
\hline M.K. & 12 & $37,5 \%$ & 6 & $18,75 \%$ & 1 & $3,12 \%$ & 13 & $40,62 \%$ & 32 \\
\hline I.J. & 12 & $42,86 \%$ & 4 & $14,28 \%$ & 1 & $3,57 \%$ & 11 & $39,28 \%$ & 28 \\
\hline M.B. & 16 & $41,02 \%$ & 1 & $2,56 \%$ & 8 & $20,51 \%$ & 14 & $35,9 \%$ & 39 \\
\hline
\end{tabular}

[4] Nei discorsi analizzati, si trovano forme di deissi personale, spaziale, temporale, del discorso, ma non di deissi sociale. Le forme di deissi reperite non si differenziano solamente da parlante a parlante, ma anche in base alla situazione. 
Osservando la tabella (tab.1) notiamo che nel discorso di Kujundžić la maggioranza delle deissi è costituita dalle deissi del discorso che lo rendono alquanto circoscritto, programmato e coeso, e dà l'impressione di non aver lasciato niente in sospeso. La parola "dakle" (it. dunque), ripetuta 5 volte, fa supporre che ci sia una logica conclusione a ogni argomento iniziato. Le deissi di persona presentano una polarizzazione o un distacco, tra la prima persona singolare " ja” (it. io), "mene" (it. me) e la terza plurale "oni" (it. loro), "one" (it. loro, f.) e differenzia quello che fa lui personalmente e quello che fanno o hanno fatto gli altri politici. Nel testo sono presenti 6 deissi di luogo, inserite per ricordare al pubblico bosniaco l'appartenenza a un territorio comune, in questo caso anche familiare. Mostar, il luogo in cui ha sede la campagna politica, è anche il luogo dove si trova la tomba del nonno, coincidenza dalla quale deve trarre il maggior vantaggio possibile, magari calcando sul momento emotivo e patriottico. Il discorso di Ivo Josipović presenta meno deissi degli altri soggetti indagati, e per lo più sono quelle del discorso, seguite da deissi di persona, di luogo e solo qualche deissi temporale. Qui troviamo una tripartizione del discorso espresso nella prima persona singolare/plurale: “ja” (it. io), "svoje” (it. mio), "moj" (it. mio), "moji" (it. miei), "naš" (it. nostro), nella seconda plurale; "vi" (it. voi), "vaš" (it. vostro) e nella terza plurale "oni", "im" (it. loro, a loro).

Josipović si collega con i suoi ascoltatori e li include nel suo discorso come collaboratori diretti, persone che lo conoscono, per cui non si sofferma in approfondimenti di carattere personale. Le deissi del discorso vengono usate solo per i collegamenti indispensabili alla stesura del testo orale. Anche se la situazione elettorale implica un discorso formale, Bandić non rinuncia alla familiarità con i suoi concittadini, al suo modo fraterno di porsi e vive la sua città (Zagabria) come parte di sé. Nella categoria della deissi spaziale c'è solo una voce $(t u)$, in quanto nel nome della sua città è incluso tutto il suo operare, ma anche l'essere.

L'uso delle deissi nei tre discorsi esaminati ci fa capire le relazioni presenti tra parlanti e ascoltatori, la dipendenza del discorso dal contesto, ma anche il carattere stesso della persona. La presentazione di sé (selfpresentation) e la narrazione di fatti concernenti la propria vita privata (self-disclosure), come già rilevato da Johansson (2008) e Langer (2010), sono oggetti di interesse pubblico e tratti comuni accettati nei discorsi 
politici in generale e non potevano mancare anche in questa indagine. Lo stile prescelto è caratterizzato dallo sforzo di costruire dapprima positive immagini di sé, e secondariamente di denigrazione degli avversari politici. Il discorso di Josipović è grammaticamente il più corretto e circoscritto e la sua esposizione orale è lineare e più simile a una ipotetica falsariga in versione scritta. L'uso di un basso numero di espressioni deittiche aumenta l'esplicitezza e sequenzialità nella struttura. Il soggetto con il maggior numero di deissi è Bandić con un discorso personale, informale e implicito. Tra i due estremi troviamo Kujundžić, ricco di elementi evocazioni ed elementi retorici che colpiscono la sfera emotiva.

3.2. Nell'analisi dell'enunciato implicato, è centrale e non trascurabile il concetto di implicatura, visto che si pone come esempio paradigmatico della natura e del potere delle spiegazioni pragmatiche dei fenomeni linguistici. È possibile dimostrare che le origini di questo tipo di inferenza si trovino esternamente all'organizzazione della lingua, nei principi generali che regolano l'interazione comunicativa (Levinson, 1985; Yule, 1986; Papi, 2000). L'implicatura conversazionale parte dalla constatazione che nella lingua è possibile capire più di quanto viene detto, per cui non ci si perde in esplicitazioni ampie su elementi noti ai dialoganti. La metafora è la forma estrema, lo sfruttamento massimo dell'implicatura conversazionale e nasce dalla teoria del paragone, quando si cancellano le similitudini. Secondo Grice (1975), l'implicatura sorge quando viene violata una delle massime: di qualità, di quantità, di relazione e di modo. Oggi è risaputo che la violazione delle massime è più frequente di quanto si pensi ed è quasi una norma, non un'eccezione.

Nel discorso di Kujundžić abbiamo trovato quattro implicature conversazionali (un numero piuttosto ridotto un discorso politico). Esse sono sempre dipendenti dal contesto e nascono in un ambiente ristretto, circoscritto, tra persone che si conoscono. Le implicature in questo discorso vengono utilizzate dal parlante, perché vuole dimostrarsi vicino al pubblico e ai futuri elettori: na domaćem terenu (it. a casa), una specie di metafora che indica la vicinanza con il pubblico, l'essere "a casa". Un'altra metafora usata dal politico è la parola "kosti" (it. ossa) che rappresenta la vita e la morte, ma anche una provenienza e un'appartenenza emotiva e culturale alla comunità croata della città di Mostar. Nell'enunciato "Postoje 
dublje veze, stvarne veze" (it. Esistono legami più profondi, legami veri) viene violata la massima di modo, in quanto non esprime in maniera chiara di quali rapporti si tratti. Il significato reale delle sue parole non è rintracciabile per chi non conosce nel dettaglio il contesto socioculturale. "Dvije lutke na papiru" (it. Due bambole su carta) è un'espressione con la quale si cerca di far capire gli errori commessi da due politici che lo hanno preceduto.

L'inserimento di elementi metaforici nel testo di Ivo Josipović avvicina l'oratore pubblico facendolo sentire parte integrante di quell'ambiente. Il suo discorso ben strutturato, lineare, viene arricchito da tre implicature inserite alla fine di una sequenza retorica e impegnativa: "Ribu smo čistili i čistit ćemo od glave" (it. Il pesce l'abbiamo pulito, e lo faremo ancora partendo dalla testa) "Mačka iz političkih vreća" (it. A scatola chiusa) e "Napravili smo važne korake" (it. Abbiamo fatto passi in avanti). La prima sottolinea l'impegno profuso fino a ora per risolvere alcuni problemi politici; la seconda simboleggia una ribellione verso modelli elettivi nulli e inefficaci; mentre l'ultima non spiega, a una prima lettura, quali siano i passi fatti di cui parla. Soltanto chi conosce e segue giornalmente la realtà croata lo può intendere. Sono implicature inserite in posti strategici del discorso: danno l'opportunità a chi ascolta di fare un breve stacco mentale, una pausa che allenterà lo sforzo dettato dall'ascolto prolungato di un discorso strutturato.

Bandić usa una similitudine, "Otpasti ko jelenji rogovi" (it. Perdere come il cervo perde le corna) che, pur non essendo un'implicatura (visto che conserva l'elemento di similarità: $k o$, ossia come), offre un'immagine traslata densa e pregnante. Dall'implicatura "Milan Bandić" è possibile capire più di quanto sia detto: probabilmente si vuole sottolineare il fatto che la prima parte del nome del suo partito (Milan Bandić 356, Stranka rada i solidarnosti - Milan Bandić 356, Partito del lavoro e della solidarietà), ossia quella che porta il suo nome, un giorno verrà dimenticata. Rimarrà, invece, la fondatezza sul lavoro attivo per ben 365 giorni all'anno.

L'analisi delle implicature ci porta a concludere che le loro funzioni in ciascun parlante sono differenziate: Kujundžić le usa per risvegliare emozioni, Josipović per alleggerire il discorso, Bandić per lo stile assolutamente colloquiale. Difatti ne usa una sola, probabilmente perché 
il suo discorso contiene già parecchie altre forme implicite e un'ulteriore aggiunta di metafore renderebbe il testo troppo implicito e di difficile comprensione, nonché troppo informale.

3.3. Un interesse particolare è stato rivolto alle spiegazioni funzionali dei tratti linguistici in quanto la struttura della lingua non è indipendente dagli usi cui essa è preposta, e può dare spiegazioni funzionali forti dei fenomeni linguistici facendo riferimento ai principi pragmatici. L'analisi "dell'unità di comunicazione linguistica che il parlante può produrre con un'unica e precisa interazione e senza che vi si frapponga l'intervento dell'ascoltatore" (Berruto, 1974, 83), ossia dell'atto linguistico, può dare interessanti informazioni sul parlato dei politici in virtù della forza intrinseca che lega dire e fare. Secondo Austin (1987), chi parla non si limita a produrre enunciati, ma esegue anche atti, grazie a questa forza a essi associata per convenzione. Seguendo la classificazione di Searle (1975), nella tabella (tab. 2) rileviamo i dati dell'analisi degli atti linguistici individuati.

Tabella 2. Atti linguistici nel discorso dei tre partecipanti

\begin{tabular}{|c|c|c|c|c|c|}
\hline & A. commissivi & A. direttivi & A. rappresentativi & A. espressivi & Tot. \\
\hline M.K. & 1 & 1 & 21 & 2 & 25 \\
\hline I.J: & 4 & 2 & 20 & 2 & 28 \\
\hline M.B. & 1 & 1 & 20 & 11 & 33 \\
\hline
\end{tabular}

Sono solo due gli atti commissivi e direttivi usati da Kujundžić. Ciò sta a indicare che egli, da parlante, non vuole ingolosire la gente con promesse da parte sua, né pretende che il popolo faccia qualcosa per lui, ossia che prenda posizione a suo favore. Si potrebbe trattare di un atteggiamento rispettoso, oppure essere indice di superficialità politica, di scarsa programmazione, di poca esperienza politica o insicurezza. L’atto commissivo usato è il seguente: "Ja ću učiniti da taj narod u Bosni i Hercegovini neće biti poniženi" (it. Io farò in modo che questo popolo della Bosnia ed Erzegovina non venga più umiliato) e quello direttivo Svakim danom ćete nešto upoznavati o meni (it. Ogni giorno scoprirete qualcosa su di me). Dato che il discorso si è tenuto in una città a lui cara, è comprensibile un certo numero di atti rappresentativi (21) legati alla città 
di Mostar, alla sua vita passata e presente: "U Mostaru sam, već od '43. a rođen sam '57." (it. Sono a Mostar dal '43, ma sono nato nel '57); "Tu negdje su kosti moga dida Marka" (it. Qui da qualche parte giaciono le ossa di mio nonno Marko); "Ja već 15 godina svake godine dolazim u Mostar kao profesor" (it. è da 15 anni che vengo a Mostar come docente tutti gli anni); "Proizveo sam mnoge koje sam učio ovdje", (it. Ho formato molte persone a cui ho insegnato qui), ecc. Solo due gli atti espressivi "Želim se zahvaliti" (it. Desidero ringraziare), "Na što sam ponosan" (it. Ne sono fiero).

Nel discorso di Josipović troviamo il numero più alto di atti commissivi: "Želim ostvariti vlast građana za građane i u rukama građana" (it. Desidero dare il potere dei cittadini ai cittadini, metterlo sulle loro mani); "Prilažem stoga novi izborni model" (it. Propongo un nuovo modello di votazione); "predlažem da se Ustavom uvede model preferencijalnog glasovanja i izravnog izbora zastupnika" (it. Propongo che la Costituzione introduca il modello preferenziale e di scelta diretta dei rappresentanti); "Ribu smo čistili i čistit ćemo od glave" (it. Il pesce lo abbiamo pulito e lo puliremo dalla testa). Ciò potrebbe significare che si tratti di un politico esperto che sa come dirigere e pianificare, che coinvolge i suoi ascoltatori dando loro fiducia e stima. Usa due atti direttivi: "Mogu li još jednom tražit vaše povjerenje" (it. Posso chiedere la vostra fiducia ancora una volta); "Građani bi birali više od pola sastava parlamenta" (it. I cittadini sceglierebbero più della metà del Parlamento), e due espressivi, quello del ringraziamento iniziale riguardante la sua precedente carriera e il supporto del suo pubblico: "Ponosan sam" (it. Sono fiero); "Vjerujem da možemo, znamo i moramo bolje" (it. Credo che possiamo, sappiamo e dobbiamo fare del nostro meglio). Ovviamente, anche il suo discorso è profuso di atti rappresentativi: "U politici je povjerenje sve (it. In politica la fiducia è tutto), "Pravda je u srcu" (it. La giustizia sta nel cuore), "To je naš stari osjećaj za narodnu pravicu" (it. Questo è il nostro senso remoto di giustizia popolare), "To je koncept razvoja društva" (it. Questa è l'idea di sviluppo della società), "2010 godine u Ustav Republike Hrvatske uvesti odredbu o nezastarijevanju ratnog profiterstva i pretvorbenog kriminala" (it. Nel 2010, la Costituzione della Repubblica di Croazia introduce una disposizione sul divieto di estinzione dei reati commessi per interessi e scopi di lucro nel periodo della guerra e del crimine della privatizzazione); "Građani s 10.000 potpisa mogu 
staviti svaku temu na dnevni red sabora $80 \%$ građana želi birati zastupnike u saboru imenom i prezimenom" (it. Con 10.000 firme i cittadini possono mettere qualsiasi punto all'ordine del giorno del Parlamento qualsiasi l'80\% dei cittadini desidera eleggere i rappresentanti del Parlamento per nome e cognome); "Moji politički konkurenti ponašaju se kao da smo politički neprijatelji" (it. La mia concorrenza politica si comporta come se fossimo nemici politici); "Oni na prekrasnom hrvatskom trgu vide duhove i danju i noću" (it. Loro sulla bellissima piazza croata vedono gli spiriti di giorno e di notte).

Il discorso di Bandić si è svolto dopo la vittoria alle elezioni di sindaco e questo fa sì che usi un maggiore numero di atti rappresentativi. Le sue mosse sono generalmente brevi, incisive, regolarmente accompagnate da verbi: "Zagreb ne trpi isključivost" (it. Zagabria non sopporta l'esclusività); "Zagreb ne trpi negaciju" (it. Zagabria non sopporta la negazione); Zagreb ne trpi destrukciju (it. Zagabria non sopporta la distruzione); "Zagreb je konstruktivan" (it. Zagabria è costruttiva); "Zagreb je pozitivan" (it. Zagabria è positiva). L'uso di paratassi, con poche subordinate e frequenti ripetizioni di termini, facilitano la comprensione e la memorizzazione dei concetti. Gli atti rappresentativi vengono usati per constatare la sua vittoria e per dare agli ascoltatori un'idea del percorso fatto: "Ne radi toga dal će Milan Bandić pobijedit s ekipom ili ne" (it. Non per il fatto se Milan Bandić vincerà con il suo team o meno); "To nije Zagreb" (it. Questa non è Zagabria); "Ja sam bio primoran osnovati stranku" (it. Io ho dovuto fondare un partito); "Ja bih najviše volio da nemam stranke" (it. Avrei preferito non avere partiti). Il suo lungo mandato alla carica di sindaco di Zagabria fa sì che nel discorso usi parecchi atti espressivi: "Ja sam čovjek širine" (it. Io sono un uomo di spessore); "Kako je lijepo vidjeti vas opet" (it. Come è bello rivedervi); "Prekrasno" (it. Bellissimo); "Emocionalno nam je" (it. Ė emotivo); "Želim se prije svega zahvalit" (it. Desidero prima di tutto ringraziare); "Prvo želim se zahvaliti” (it. Prima di tutto desidero ringraziare); "Teško je ostati ravnodušan" (it. Ė difficile rimanere indifferente); "Teško je ostati da nije čovjek emotivan" (it. Ė difficile rimanere senza emozioni); "Ja sam čovjek od krvi i mesa" (it. Io sono un uomo di carne ed ossa); "Ne udvaram se" (it. Non vi voglio conquistare); "Nisam lažno skroman" (it. Non sono un finto modesto); "Govorim trijezan" (it. Parlo da sobrio). Ricordiamo ancora un 
unico atto commissivo "Imena luda nalaze se svuda, to ćemo ukinut" (it. I nomi degli stolti si trovano ovunque, annulleremo questa pratica) e uno direttivo "Moj savjet njima da to nikad ne rade" (it. Il mio consiglio a loro di non farlo mai).

\section{Conclusione}

La presente ricerca è circoscritta alla descrizione di tre discorsi di altrettanti uomini politici croati, condotta da una prospettiva pragmatica. Le nostre conclusioni, ancor più in quanto quantitative, sono fondate solo ed esclusivamente a partire da un discorso per politico, dalla durata inferiore ai tre minuti. In questo senso riteniamo questa ricerca un lavoro pilota per una più approfondita analisi futura. L'analisi pragmalinguistica del discorso politico ha rivelato delle differenze linguistiche di ciascun personaggio politico sottoposto ad analisi, nel loro modo di essere, di pensare e di comunicare con gli altri. D'altro lato, l'aver riscontrato una gamma di caratteristiche in comune, fa sì che questa ricerca possa allinearsi alle ricerche sul linguaggio della politica fatte precedentemente. Sul versante dei contenuti analizzati, troviamo in tutti argomenti connotati emotivamente e legati a valori popolari condivisi, come la famiglia e la patria. Similmente si nota un ricorso costante alla dicotomia di categorie contrarie e complementari (bene vs. male, vero vs. falso) dove l'io o il noi fa parte della sfera positiva e il lui e loro a quella negativa. Tra le strategie comunicative usate in tutti e tre i candidati troviamo il tono colloquiale atto a stabilire legami emotivi con il proprio pubblico, la costruzione discorsiva semplice, ideale per un linguaggio orale che tende a essere incisivo. In tutti e tre i candidati si nota un cospicuo utilizzo di atti linguistici referenziali all'indicativo presente, passato e futuro che comunicano certezze e competenze. La presentazione di sé (self-presentation) e la narrazione di fatti concernenti la propria vita privata (self-disclosure) come già rilevato da Johansson (2008) e Langer (2010) sono oggetti di interesse pubblico e tratti comuni e accettati nei discorsi politici in generale e non potevano mancare anche in questa indagine. Lo stile prescelto è caratterizzato dallo sforzo di costruire dapprima positive immagini di sé, e secondariamente di denigrazione degli avversari politici. 
Kujundžić lascia un'impressione superficiale di familiarità e amicizia, evocando fatti accaduti nella storia che lo riguarda, suscitando e mantenendo l'interesse degli ascoltatori su un piano evocativo e nostalgico. Qui possiamo liberamente parlare di personalizzazione della politica, una tendenza crescente di porre l'attenzione verso il politico, piuttosto che verso il programma e/o l'ideologia di cui è portavoce. Tale processo si è sviluppato, probabilmente, dagli interessi degli elettori che tenderebbero ad assegnare maggiore credibilità e consenso a coloro che parlano di sé, mostrandosi "emotionally closer to, and more like ordinary people" (Langer, 2010, 61). In questo testo, Kujundžić non si dimostra come "leader" in quanto, oltre a esporre la propria storia personale, non espone alcun piano politico generale. I suoi segnali discorsivi come dakle (it. dunque), znači (it. quindi), che contribuiscono alla costruzione del discorso sembrano mascherare la debolezza dell'argomentazione.

Josipović mantiene il discorso lineare e chiaro, paragonabile alla langue, al sistema linguistico, in parte aulico e accademico. Le sue frasi sono brevi e concise, tutte rispettano il canone soggetto + verbo + oggetto per cui non necessita un numero elevato di deissi. Sembra essere conscio del suo linguaggio elegante, che sa di scritto, usa due metafore solamente per avvicinarsi al pensiero, ai costumi e alle parole delle masse che lo ascoltano. Ciò potrebbe essere indice di un'esperienza politica precedente, implicando una stima per chi lo ascolta. Comunque, anche i suoi scopi comunicativi manifestano uno sforzo per convincere il pubblico facendo appello in vari modi alla fattualità, rinunciando a momenti di emotività.

Il discorso di Bandić non fa parte delle elezioni presidenziali, ma è quello espresso dopo la vittoria alle elezioni comunali. Questa differenza di contesto ha dato risultati diversi rispetto agli altri due discorsi analizzati. Egli si sente sicuro di sé, si trova a suo agio tra la gente che lo ha eletto sindaco e ne prova un certo piacere. Non ha bisogno di fare discorsi formali, anzi, abbassa la complessità concettuale della comunicazione in favore dell'emotività diretta. In questo modo aumenta l'ambiguità a discapito della precisione dei concetti. Nel linguaggio di Bandić e Kujundžić è presente un senso di orgoglio, una capacità di suscitare in chi li ascolta un'intensa emozione e una partecipazione sul piano affettivo. Questo si realizza anche grazie ai pronomi personali utilizzati: quando ricorrono 
all'io, lo fanno per parlare di caratteristiche private, di aspetti relativi al loro sé non istituzionale. L'uso della terza persona è inteso in senso per lo più negativo e il parlante ha un unico scopo: quello di distanziarsi dai suoi avversari o predecessori in quanto inadeguati per questo ruolo politico.

\section{BIBLIOGRAFIA}

ARCHER, R. L., COOK, C., 1986, Personalistic Self-Disclosure and Attraction: Basis for Relationship or Scarce Resource, in "Social Psychology Quarterly", 49(3), 268-272.

AUSTIN, J. L., 1987, Come fare cose con le parole, Martinetti, Genova.

BERRUTO, G., 1974, La sociolinguistica, Zanichelli, Bologna.

BONGELLI, R., RICCIONI, I., VIGGIANO, L., LO BUE, S.,

CAMPUS, D., 2010, Mediatization and Politics in Italy and France: The Cases of Berlusconi and Sarkozy, in "International Journal of Press/ Politics", 15(2), 219-235.

CEDRONI, L., 2014, Politolinguistica. L'analisi del discorso politico, Carocci Editore, Roma.

COLLINS, N. L., MILLER, L. C., 1994, Self-disclosure and liking: A metaanalytic review, in "Psychological Bulletin", 116 (3), 457-475.

CORTELAZZO, M. A., 2016, Il linguaggio della politica, Accademia della Crusca/Editoriale L'Espresso, Firenze/Roma.

DELL'ANNA, M. V., 2010, Lingua italiana e politica, Carocci, Roma.

DESIDERI, P., 1983, Il discorso politico: ambiti metodologici, modalita' e istanze enunciative, luoghi argomentativi, in "Bloc Notes" vol. 6, 23-42.

DESIDERI, P., 1998, Il discorso politico tra pragmatica e argomentazione, in "List. Quaderni di studi linguistici", 71-92, EUROMA, Roma.

DESIDERI, P., 1998b, Il discorso politico da Mussolini a Bossi, in BORELlO, E., PETROLINO, A., VEZZANI, A., Miti, consumi e sogni tra comunicazione di massa e marketing, 73-91, Sintagma Editrice, Torino. 
GIANSANTE, G., 2011, Le parole sono importanti. I politici italiani alla prova della comunicazione, Carocci, Roma.

GILBERT, D., CAMBON, L., 2003, Paradigms of the Sociocognitive Approach, in DUBOIS, N., (ed.), A sociocognitive approach to social norms, Routledge, London, 123-147.

GRICE, H. P., 1989, Logic and Conversation, in COLE, P., MORGAN, J., (eds.), Syntax and Semantics, vol. 3, Academic Press, New York, 22-40.

JOHANSSON, M., 2008, Presentation of the Political Self: Commitment in Electoral Media Dialogue, in "Journal of Language and Social Psychology", 27(4), 397-408.

LANGER, A. I., 2010, The Politicization of Private Persona: Exceptional Leaders or the New Rule? The case of the United Kingdom and Blair Effect, in "International Journal of Press/Politics", 15(1), 60-76.

LASSWELL, H. D., FOX, M. B., 1979, The Signature of Power: Buildings, Communication, and Policy, New Brunswick, Rutgers University Press, N. $\mathrm{J}$.

LEVINSON, S. C., 1985, La pragmatica, Il Mulino, Bologna.

MARLETTI, C., 2010, La Repubblica dei Media. L'Italia dal politichese alla politica iperreale, Il Mulino, Bolgna.

PAPI, M. B., 2000, Che cos'è la pragmatica, Gruppo Editoriale Fabbri, Milano.

POGGIO, B., VALASTRO, O. M., (ed.), 2012, Le sequenze narrative nei discorsi politici: la presentazione di sé, in "Raccontare Ascoltare Comprendere M@gm@”, vol. 10 n. 1, gennaio-aprile 2012.

RUGGIANO, F., 2013, Ideologia linguistica della stampa nella campagna elettorale per le politiche del 2013, in "Circula", no.1, pp. 216-234. http:// savoirs.usherbrooke.ca/handle/11143/8001

SCHLENKER, B. R., 1980, Impression management: The self-concept, social identity, and interpersonal relations, Brooks, Monterey, CA.

SEARLE, J. R., 1976, Atti linguistici. Saggio di filosofia del linguaggio, Boringhieri, Torino. 
SPINA, S., 2012, Openpolitica. Il discorso dei politici italiani nell'era di Twitter, Franco Angeli, Milano.

STANYER, J., 2007, Modern Political Communication, Polity, Cambridge. TORZI, I., 2006, Appunti di pragmalinguistica, I.S.U. Università Cattolica, Milano.

VAN DIJK, T., 2005, War rhetoric of a little ally. Political implicatures and Aznar's legitimatization of the war in Iraq, in "Journal of Language and Politics", 4(1), 65-91.

WODAK, R., 2009, The discourse of politics in action. Politics as Usual, Macmillan, Palgrave.

YULE, G., 1986, Pragmatics, Oxford University Press, New York.

ZUCZKOWSKI, A., 2012, Le sequenze narrative nei discorsi dei politici: la presentazione di sé, in "m@gm@”, Rivista Internazionale di Scienze umane e Sociali, v.10 n.1, http://webcache.googleusercontent.com/ search?q=cache:RvoPd4xk7MEJ:www.magma.analisiqualitat.

ZUPNIK, Y. L., 1994, A pragmatic analysis of the use of person deixis in political discourse, in "Journal of Pragmatics", 21(4), 339-383. 


\section{THE PRAGMALINGUISTIC ANALYSIS OF THREE SPEECHES GIVEN BY CROATIAN POLITICIANS}

Although directed towards immediacy, simplicity and clarity, the political discourse hides generic concepts and clichés. It often appears unclear, vague, ambiguous, and self-referential. In this type of discourse pragmalinguistic research identifies relevant information related to all the implicit elements of the context and co-text. In this research, we analyzed and discussed three speeches given by Croatian politicians. All the similarities and differences between them were found through the analysis of deixis, presuppositions, conversational implications and speech acts.

Keywords: pragmalinguistics, language of politics, speech acts, conversational implicatures, deixis

\section{PRAGMALINGVISTIČKA RAZMATRANJA O TRIMA DISKURSIMA HRVATSKIH POLITIČARA}

Politički diskurs, premda teži neposrednosti, jednostavnosti i jasnoći jezičnoga izraza, zapravo se krije iza stereotipnih pojmova i klišeja. Govor često ispada nerazumljiv, nejasan, dvosmislen, samoreferencijalan. Pragmalingvistička istraživanja pronalaze u takvim diskursima relevantna saznanja vezana za sve implicitne oznake konteksta i koteksta. U ovome smo istraživanju analizirali i potom raspravili govor triju hrvatskih političara, a sve sličnosti i razlike u govoru proizlaze iz analize deikse, presupozicija, implikatura i govornih činova.

Ključne riječi: pragmalingvistika, jezik politike, govorni činovi, implikature, deikse 